
Original
Article

\title{
Curcumin Attenuates Apoptosis and promotes cell proliferation and Protects Rats against Gentamycin-induced Nephrotoxicity
}

\author{
Mohamed Mohamed Abdelmoaty and Reda Abdelnasser Imam
}

Department of Anatomy, Faculty of Medicine, Cairo University, Egypt

\begin{abstract}
Background: The aminoglycoside antibiotic gentamicin (GEN) was associated with nephrotoxicity in humans. Curcumin (CUR) supplementation is effective for preventing or ameliorating the renal toxicity of some drugs in patients and animals. Aim of Work: Investigating the possible protective role of Curcumin against the adverse gentamycin renal changes in rats and ellucidating its mechanism of action.

Material and Methods: Twenty four adult male rats were used in this study. The animals were divided into four groups; Group I (control group), GEN group (II) received Gentamicin (100 mg/kg/day) for 21 days, CUR group (III) received Curcumin for 21 days, GEN + CUR group (IV) received GEN together with CUR (100 mg/kg) for 21 days. All animals were euthanized at the end of experiment and the kidney was obtained. Renal homogenates were assessed for oxidative stress markers Glutathione (GSH) and Malodialdehyde (MDA). Paraffin sections were processed then stained with Hx \& E, Masson's trichrome and immunohistochemical stains: BAX (for apoptosis) and PCNA (for cell proliferation).

Results: Curcumin had significantly $(p<0.05)$ improved the level of MDA and GSH in renal tissue as compared to Gentamycin received rats. Histologically, gentamycin adverse histological changes were markedly improved in GEN + CUR group. The area $\%$ of collagen fibers and positive BAX reaction in GEN+ CUR group was significantly decreased $(p<0.05)$ as compared to GEN group. The area \% of positive PCNA reaction in GEN+CUR group had increased significantly $(p<0.05)$ as compared to GEN group.
\end{abstract}

Conclusion: Curcumin could attenuate apoptosis, promote cell proliferation and protect rats against Gentamycin nephrotoxicity.

Received: 15 Oct 2018, Accepted: 09 Feb 2019

Key Words: Curcumin, gentamicin, kidney, rats.

Corresponding Author: Mohamed Mohamed Abdelmoaty, MD, Department of Anatomy, Faculty of Medicine, Cairo University, Egypt, Tel.: +2 01008030473, E-mail: mmmotey@yahoo.com.

ISSN: 2536-9172, Dec 2018 Vol.2, No.2

\section{INTRODUCTION:}

Nephrotoxicity is the most concerned side effect attributed to aminoglycoside antibiotic therapy. In spite of their nephrotoxic reactions, aminoglycosides are widely used against Gram-negative bacteria and bacterial endocarditis. Gentamicin (GM) is the most common nephrotoxic aminoglycoside ${ }^{[1]}$. It has been estimated that up to $30 \%$ of patients treated with GM for more than 7 days show some signs of renal impairment ${ }^{[2]}$. Although the exact mechanisms of nephrogenic injury induced by aminoglycoside antibiotics are not fully understood yet, but oxidative stress induction and the impairment of intracellular organelles functionality are supposed to be involved in this complication ${ }^{[3]}$. Moreover, drug accumulation in the plasma membranes of tubular cells especially the proximal tubules might play a role in aminoglycosides-induced nephrotoxicity ${ }^{[4]}$. As confirmed by $^{[5]}$, generation of reactive oxygen metabolite (ROM) may be the basis of a variety of insults, such as that induced by gentamicin and they added that treatment with several natural and synthetic antioxidant substances has been shown to be useful in either its prevention or its amelioration in experimental rats. ${ }^{[6]}$ had tested several extracts of medicinal plants including curcumin against GM-induced nephrotoxicity in rats and they uncertainly proved the basis of the protective action of curcumin extract by their antioxidant properties). Curcumin (CUR) is described by ${ }^{[7]}$ as a major yellow pigment which is widely used as a spice and coloring agent in several foods and they mentioned the curcuminoids as the effective chemical that are responsible for the yellow color of turmeric, as well as the yellow color of curry. For their antioxidant and anti-inflammatory activities, curcumin and the other curcuminoids have been entered into Phase I clinical trials for cancer chemoprevention by the National Cancer Institute ${ }^{[8]}$. The aim of our research is to investigate the possible preventive role and to elucidate the mechanism of action of Curcumin on gentamicin.induced nephrotoxicity in order to gain new insights into the prophylaxis of gentamicin-induced nephrotoxicity which is a common problem limiting the use of this drug. 


\section{MATERIAL AND METHODS}

Twenty four adult Wistar rats, weighing 200-250 g, were utilized in this study. The experiment agreed with all ethics of animal research and was approved from local ethical committee of the faculty of medicine, Cairo University. The animals were housed at the Institute for ophthalmologic Research in Egypt under controlled conditions with the twelve-hour day/night cycle, at the temperature of $23^{\circ} \mathrm{C} \pm 2{ }^{\circ} \mathrm{C}$, and free access to food and drinking water.

\section{Chemicals:}

A. Gentamicin (GEN) sulfate, available commercially as Epigent $®(80 \mathrm{mg} / 2 \mathrm{ml}$ ampoules $)$, was obtained from the Egyptian International Pharmaceutical Industries Co. (EIPICO, 10 ${ }^{\text {th }}$ of Ramadan City, Egypt) and diluted in $10 \mathrm{ml}$ saline and as $1.2 \mathrm{ml}$ for every 100 grams rat weight.

B. Curcumin (CUR) (diferuloylmethane) [1, 7-bis (4-hydroxy-3-methoxyphenyl)-1, 6-heptadiene-3, 5- dione] was obtained as Turmeric Curcumin 300mg capsules (Thompson company, USA) and dissolved in corn oil (one capsule dissolved in 15 $\mathrm{ml}$ corn oil) and given via gastric gavage as one $\mathrm{ml}$ for every 100 grams rat weight .

All chemicals used were of good quality and highest analytical grade.

\section{Experimental Design:}

Experimental animals were randomly divided into four equal groups of 6 animals each:

Group I (control group): was used as a control group and received physiological saline solution $1 \mathrm{ml} /$ day intraperitoneally.

Group II (GEN group): was given Gentamicin intraperitoneally in a dose of $100 \mathrm{mg} / \mathrm{kg}$ body weight (BW)/24h for 21 days $^{[9]}$.

Group III (CUR group): Received CUR (200 mg/ $\mathrm{kg}$ body weight [bw] /day) dissolved in corn oil and administered to animals orally by gastric gavage for 21 days ${ }^{[10]}$.

Group IV-GEN and CUR-treated group: Received GEN (100 mg/kg bw / day) intraperitoneally for 21 days and CUR (200 mg/ kg bw /day) orally by gastric gavage for 21 days concomitantly.

Animals of all groups were treated over a period of 21 consecutive days. Following the last application, all animals were anaesthetized, euthanized by cervical dislocation and the kidneys were subsequently removed and subjected to:

\section{1- Biochemical analysis:}

\section{A. Lipid peroxidation:}

The LPO levels were measured according to the method described by ${ }^{[11]}$ using biodiagnostic kits. This method was based on estimation of the released malondialdehyde (MDA) molecules, as a result of oxidative damage of cell membranes. The concentrations of MDA were expressed as in nanomole of MDA per gram of tissue.

\section{B. Bioassay of non-enzymatic antioxidants:}

Non-enzymatic (glutathione, GSH) antioxidants were estimated in the homogenate of the kidneys of control male albino rats, those administered with repeated clinical doses of Gentamicin and those administered with repeated clinical doses of gentamicin and curcumin. The concentrations of GSH in tissue homogenates were estimated and expressed in milligrams of GSH per gram of tissue ${ }^{[12]}$.

\section{2- Histological analysis}

The kidneys were extracted, cleaned and fixed in $10 \%$ formaldehyde, dehydrated in alcohol and processed for paraffin embedding. Kidney tissue species were cut at a thickness of $5 \mu \mathrm{m}$ and stained with hematoxylin \& eosin for the study of morphological changes in the kidney and Masson for detecting the collagen fibres.

\section{3-Immunohistochemical study ${ }^{[13]}$ :}

Deparaffinized sections were mounted on positively charged slides and immunohistochemically assessed for BAX (to detect apoptosis) and proliferating cell nuclear antigen (PCNA) (to assess cell proliferation). After Heat-induced epitope retrieval (HIER) to deparaffinized Sections, Sections were peroxidase blocked. Primary antibodies were BAX (Dako, Denmark), PCNA (Santa Cruz, USA). Autostaining technique with Dako was performed handling Dako EnVision Flex/HRP as secondry antibodies. The prepared sections were examined and photographed using a Canon digital camera (Canon, Japan) attached to the IBM computer system. 


\section{Histomorphometric analysis:}

Image analysis was performed using the software Leica Quin 500, Germany. The area percent of collagen fibers in Masson's trichrome stain and that of positive BAX as well as PCNA were measured in a standard measuring frame using a magnification $\mathrm{x} 400$ by light microscopy transferred to the monitor's screen. These areas were masked by a green color using the computer system. Area percent values for each group were obtained from 5 different fields from different slides. Values were presented as a mean and standard deviation and statistically analyzed.

\section{Statistical analysis}

The area $\%$ of collagen fibres of the Masson reaction as well as the optical densities of BAX and PCNA reactions in the glomeruli, renal corpuscles and urinary in the different groups. Moreover, the values of GSH and MDA in renal tissues levels among different goups were measured in nmol/ gram tissue for MDA and in milligram/ gram tissue for GSH. All values were presented as mean and standard deviation $( \pm \mathrm{SD})$. Statistical analysis was performed using one-way analysis of variance (ANOVA) followed by post-hoc Tukey HSD test to compare variables among the different groups. A value of $P<0.05$ was con $\neg$ sidered significant. Data was tabulated and repre $\neg$ sented graphically.

\section{RESULTS}

\section{Biochemical Results:}

The activities of GSH and MDA in kidneys of groups II and IV were significantly affected by the injected dose of gentamicin. In (Table 2 and histograms 4, 5), the level of GSH was significantly increased in CUR+GM group in relation to GEN renal tissues while the level of MDA was significantly decreased in CUR + GM group in relation to group II renal tissues.

\section{Histological analysis}

Sections from control (I) group (Figure 1A) showed normal histological structure of the glomeruli and renal tubules. The fibrosis was minimal as detected by Masson's trichome stain (Figure 2A). In GEN group (Figure 1B) renal sections showed shrunken glomeruli, wide Bowman's space and inflammatory infiltrates. Proximal tubules showed necrosis, vacuolization of cytoplasm and loss of the brush border. The distal tubules were preserved. Moreover, the apoptotic bodies appeared in some specimens (Figure 1C). The fibrosis was massive as detected by Masson's trichome stain (Figure 2B). CUR group (III) (Figure 1C) showed similar histology to the control group. No noticeable fibrosis as detected by Masson's trichome stain was observed (Figure 2C). CUR and GM group (IV) (figure 1D) revealed partial improvement of the histopathological alterations induced by gentamicin in the form of normal glomeruli and space of bowman capsule, alleviated proximal tubular degeneration and mild to moderate mononuclear inflammatory infiltrates. The rest of histopathological changes produced by gentamicin were completely prevented by curcumin treatment. The fibrosis was markedly deceased as detected by Masson's trichome stain (Figure 2D).

\section{Immunohistochemical staining of $B A X$ :}

Group I: The kidneys of the rats of this group revealed negative BAX immune reaction of the tubular cell cytoplasm (Figure 3A). Group II: Revealed highly expressed reaction in the cytoplasm of the tubular cells (Figure 3B). Group III: No noticeable changes were seen to in this group in comparison with the group I (Figure 3C). Group IV: revealed apparent minimally expressed reaction in the cytoplasm of the tubular cells (Figure 3D).

\section{Immunohistochemical staining of PCNA:}

Group I: The renal cortex of the rats of this group revealed negative PCNA immune reaction of the tubular cell cytoplasm (Figure 4A). Group II: evealed apparent minimally expressed PCNA reaction in the cytoplasm of the tubular cells (Figure 4B) indicating minimal regeneration. Group III: No noticeable changes were seen to in this group in comparison with the group I (Figure 4C). Group IV: revealed highly expressed PCNA reaction in the cytoplasm of the tubular cells (Figure 4D).

\section{Histomorphometric results:}

The mean area \% of collagen fiber deposition and the optical densities of PCNA immunoreactivity and BAX immunostaining for all groups was represented in table (1) and histograms (1-3). There was a significant decrease $(P<0.05)$ in collagen fibers deposition and positive BAX immunostaining in group IV compared with group II (table 1 and histograms 1,2 ) while there was a significant increase $(P<0.05)$ in PCNA immunoreactivity in group IV compared with group II (table 1 and histogram 3 ). 

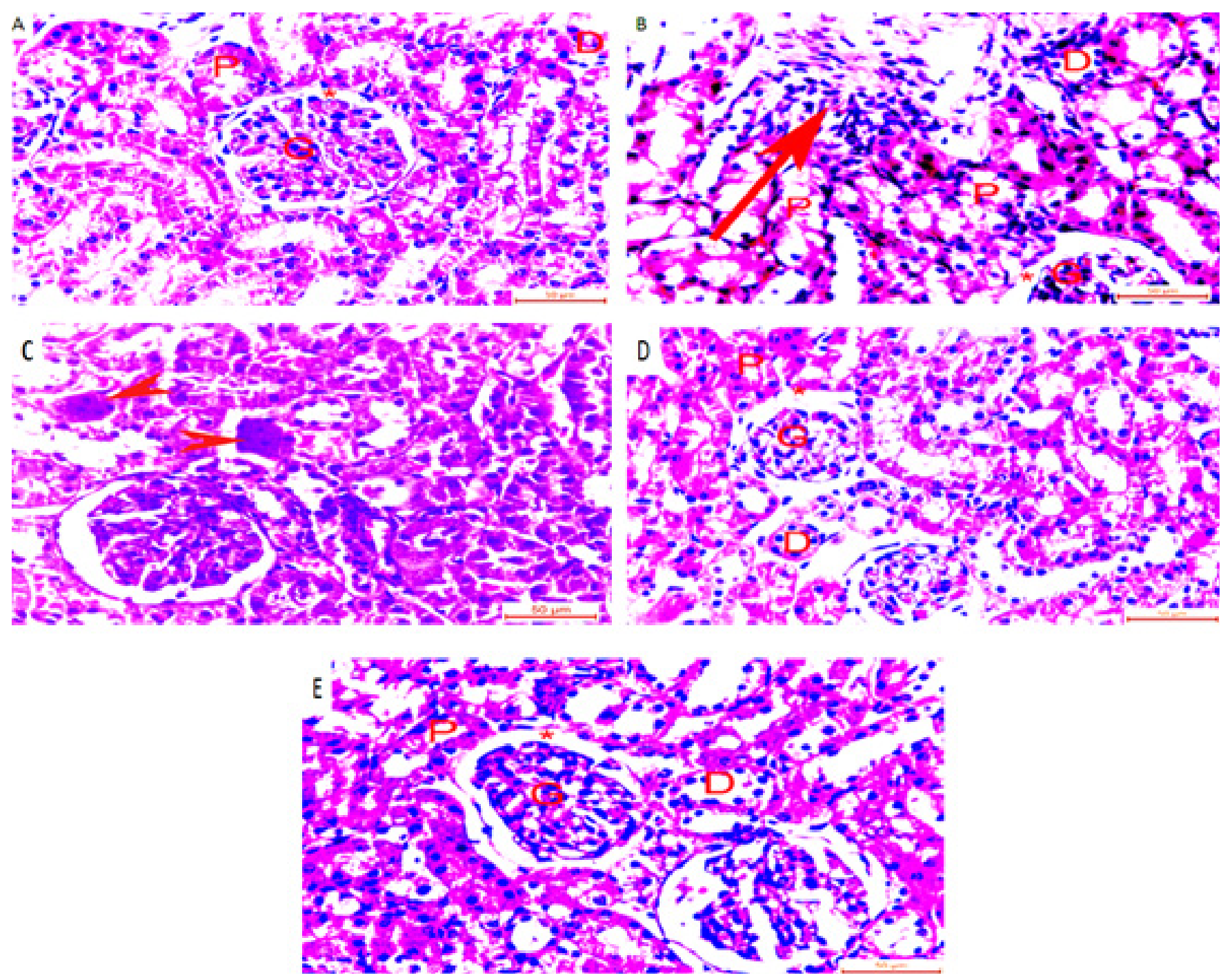

Fig. 1: A- Control group (I) showing normal glomerus $(\mathrm{G})$ and bowman capsule $(*)$. The proximal tubules $(\mathrm{P})$ appear normal with cuboidal cells having brush border. The distal tubules are also normal. B- GM group (II) showing inflammatory infiltrates (arrow), mild glomerular congestion $(\mathrm{G})$, wide bowman capsule space $(* *)$ and the proximal tubular cells $(\mathrm{P})$ appears necrotic, markedly vacuolated with loss of the brush border the distal tubules are mildly affected. C- GM group (II) showing apoptotic bodies surrounded by eosinophilic material (arrow heads). D- CUR group (III) showing normal appearance. E- GM and CUR group (IV) showing alleviation of proximal tubule changes (P) in the form of less vacoulation, little necrosis and the appearance of the lumen. The glomeruli $(\mathrm{G})$ and the distal tubules (D) appear normal.

$(\mathrm{H} \& \mathrm{E} \times 400)$ 

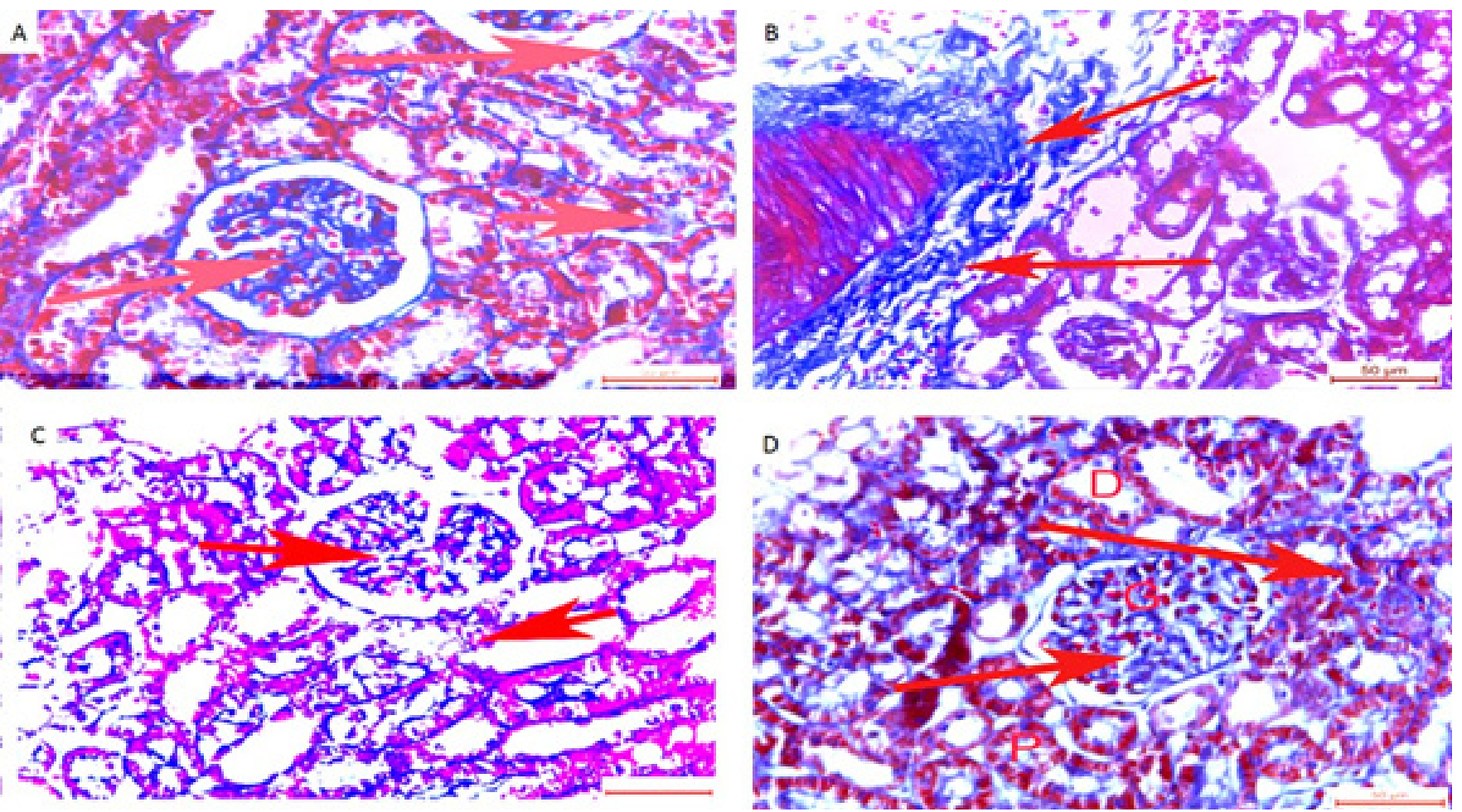

Fig. 4: A- Control group (I) showing little collagen fibres.B- GM group (II) showing massive collagen fibres deposition (arrows) in comparison to group (I).C-CUR group showing little collagen similar to the control group. D- GM and CUR group (IV) showing moderate collagen fibres deposition (arrows) in comparison to group (II).

(Masson trichrome $\times 400$ )
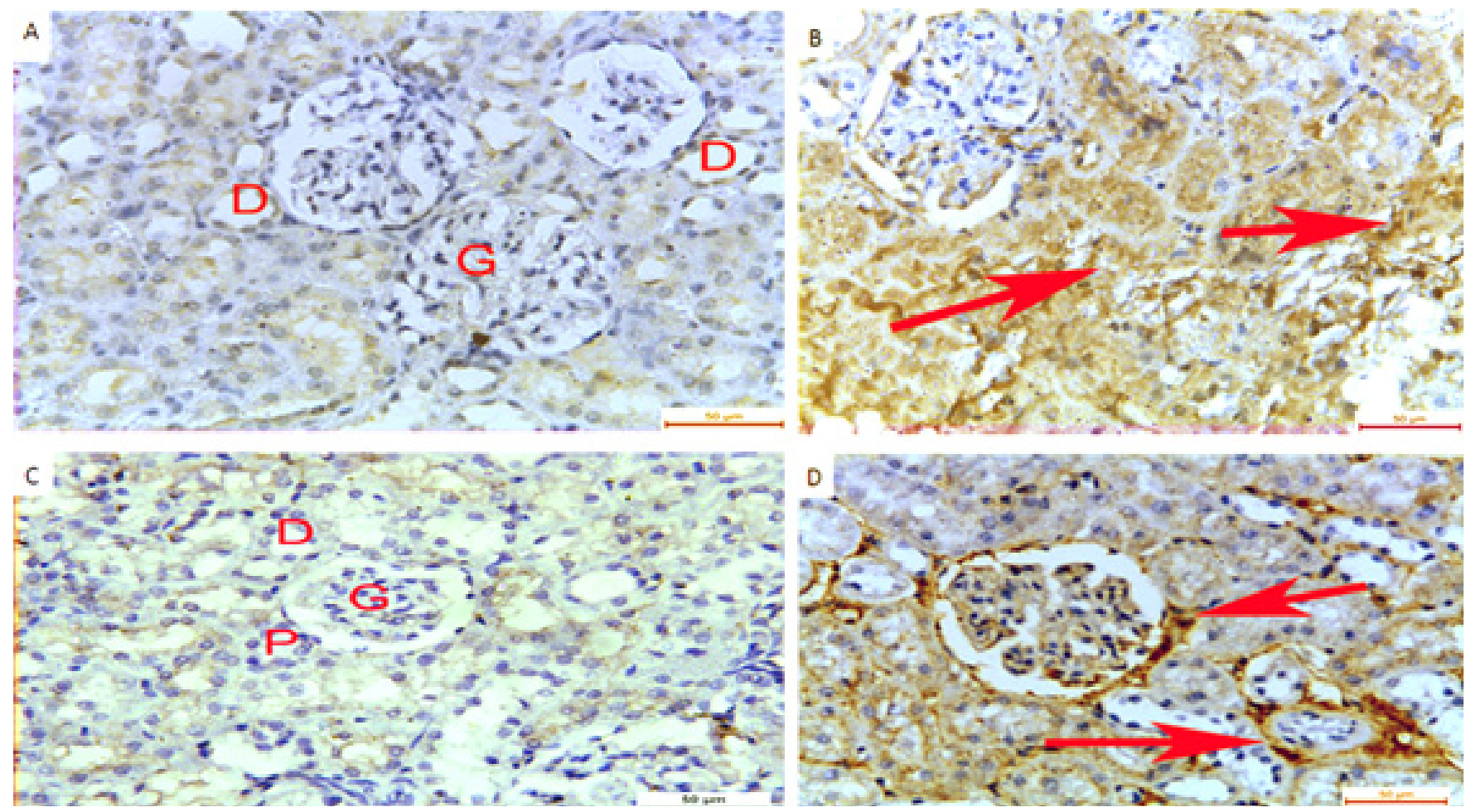

Fig. 2: A- Control group (I) showing negative BAX 2 reaction. B- GM group (II) showing high expression of BAX reaction. C- CUR group (III) showing very mild expression of BAX 2 reaction. D- GM and CUR group (IV) showing improved expression of BAX 2 reaction in comparison to group (II)

(BAX reaction $\times 400$ ) 

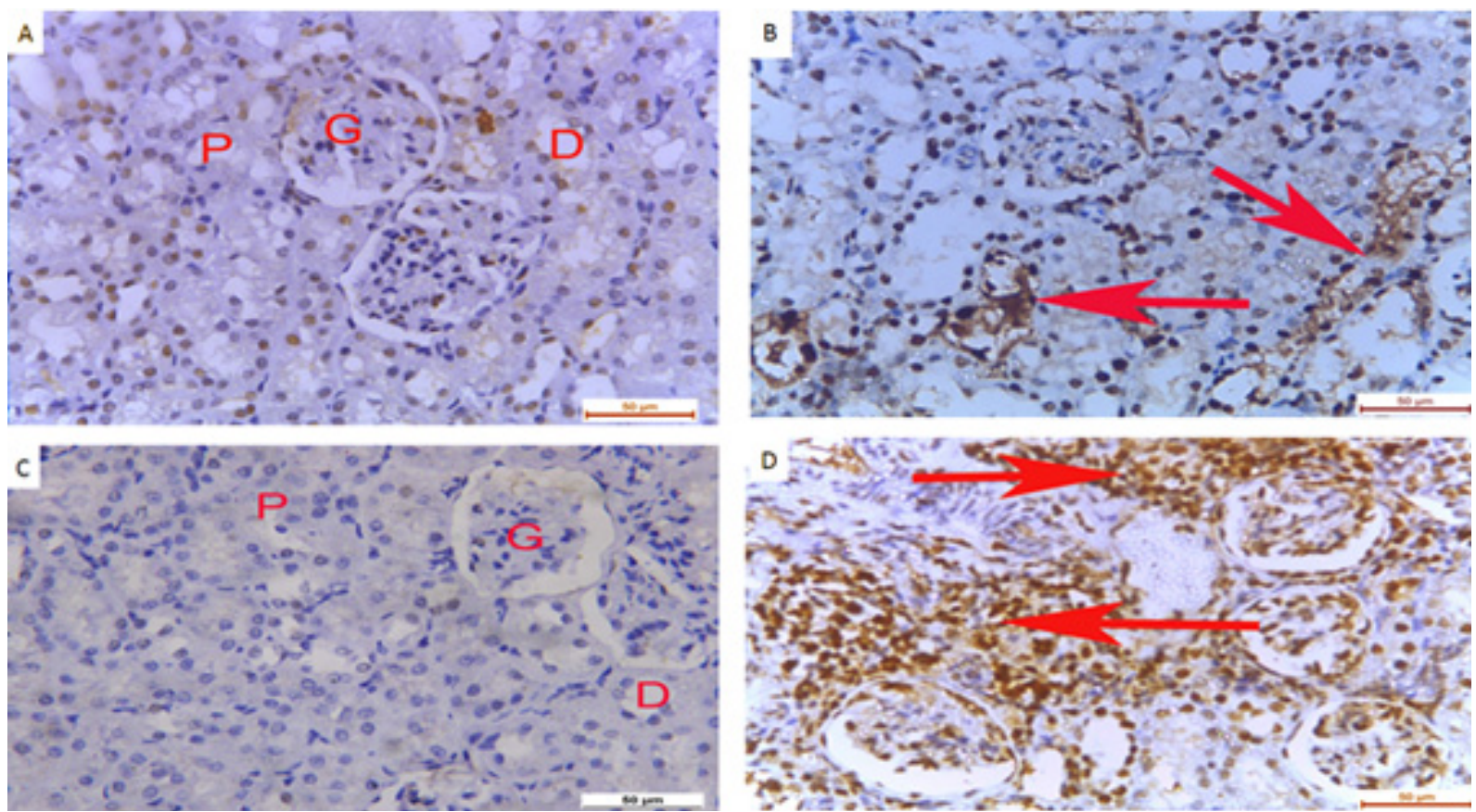

Fig. 3: A- Control group (I) showing negative PCNA reaction. B- GM group (II) showing moderate expression of pcna reaction. C- CUR group (III) showing mild expression of pcna reaction. D- GM and CUR group (IV) showing high expression of pcna reaction in comparison to group (I).

$($ PCNA reaction $\times 400)$

Table 1: Comparison of the area \% of Collagen fibers in Masson' trichrome stain positive PCNA as well as positive BAX among different groups

\begin{tabular}{lccc}
\hline & $\begin{array}{c}\text { Area \% of collagen fibers in Masson's trichrome } \\
\text { Mean } \pm \text { standard deviation }\end{array}$ & $\begin{array}{c}\text { Area \% of BAX } \\
\text { Mean } \pm \text { standard deviation }\end{array}$ & $\begin{array}{c}\text { Area \% of PCNA } \\
\text { Mean } \pm \text { standard deviation }\end{array}$ \\
\hline Control group (I) & $11.27 \pm 1.62$ & $2.50 \pm 0.65$ & $9.07 \pm 1.45$ \\
GEN group (II) & $25.08 \pm 4.03^{*}$ & $10.58 \pm 1.85^{*}$ & $12.81 \pm 2.85^{*}$ \\
CUR group (III) & $12.49 \pm 2.23^{* *}$ & $2.63 \pm 0.67^{* *}$ & $9.57 \pm 1.38$ \\
GEN+CUR group (IV) & $13.06 \pm 2.21^{* *}$ & $6.30 \pm 1.13^{*}, * *, * * *$ & $20.17 \pm 2.04 *, * *, * * *$ \\
\hline
\end{tabular}

*Statistically significant as compared to control group (group I). ** Statistically significant as compared to GEN group II. *** Statistically significant as compared to CUR group III.

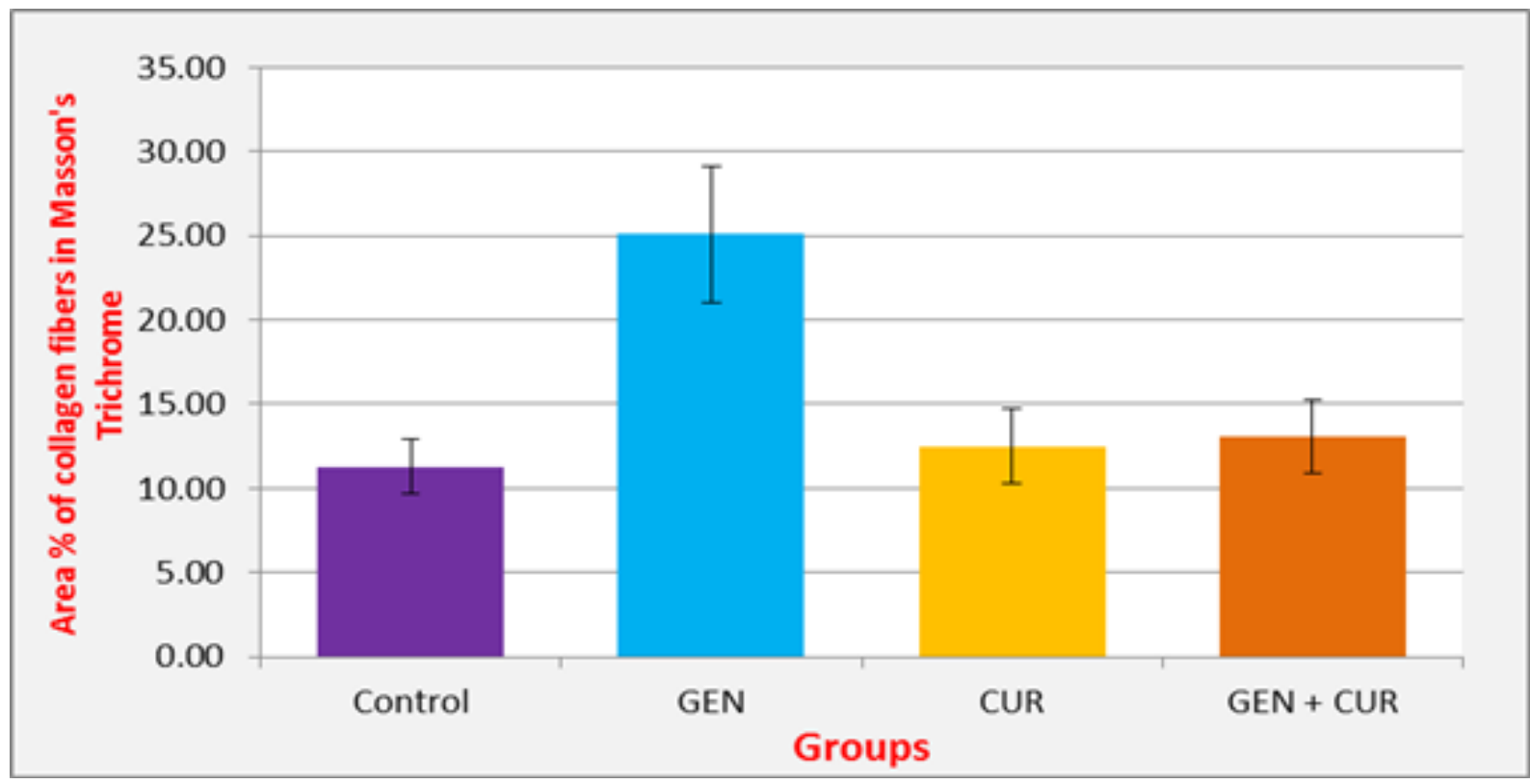

Histogram 1: Comparison of the area\% of collagen fibres between different groups 


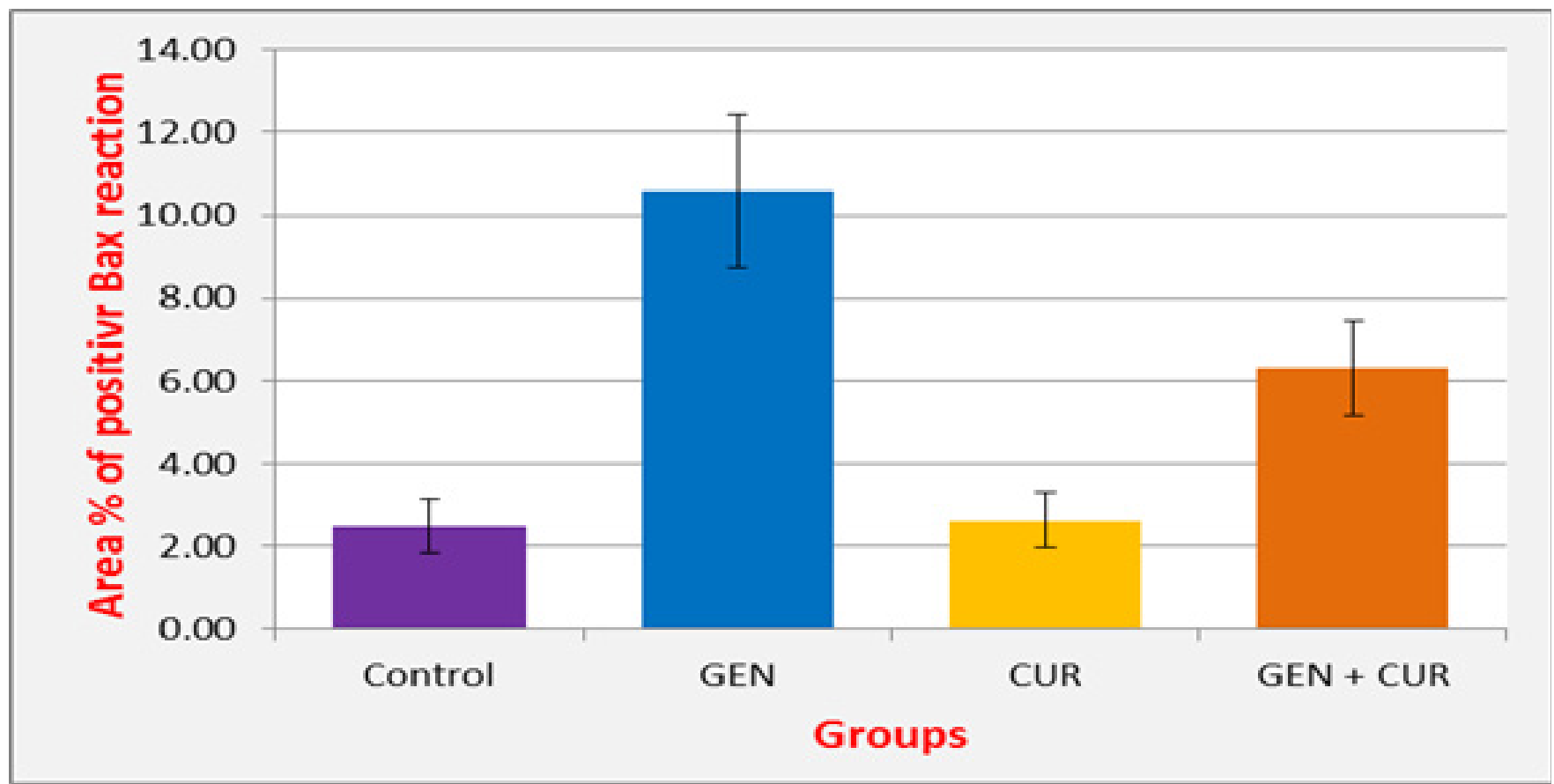

Histogram 2: Comparison of the optical density of positive BAX reaction between different groups

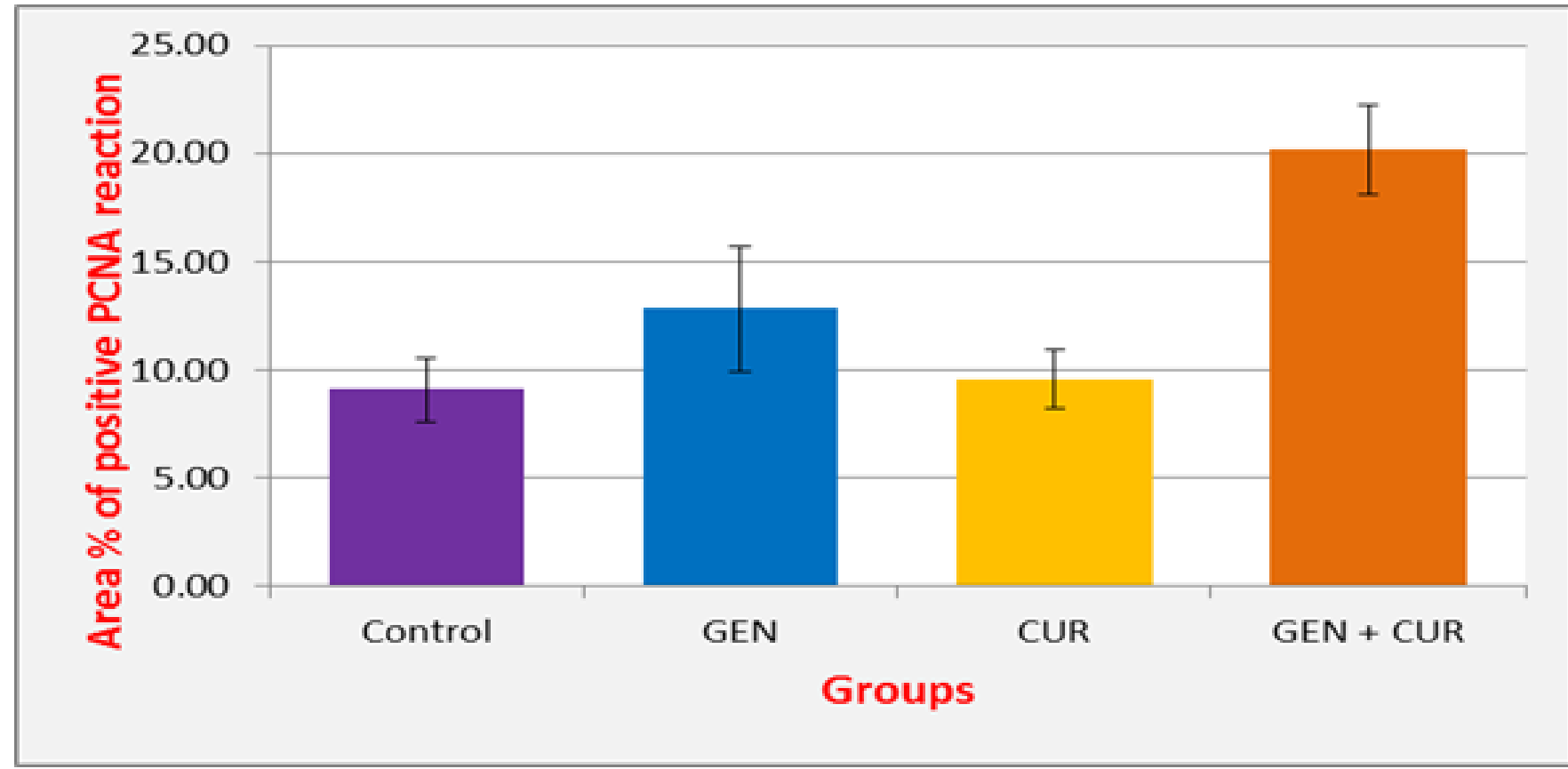

Histogram 3: Comparison of the optical density of positive PCNA reaction between different groups

Table 2: Comparison of GSH and MDA IN renal tissues levels among different groups

\begin{tabular}{lll}
\hline & GSH & MDA \\
\hline Group I & $78.77 \pm 6.70$ & $4.84 \pm 0.65$ \\
Group II & $25.80 \pm 4.76^{*}$ & $25.60 \pm 3.01 *$ \\
Group III & $73.76 \pm 13.2 * *$ & $5.05 \pm 0.76^{* *}$ \\
Group IV & $55.62 \pm 4.61 * * *, * * *$ & $11.38 \pm 1.47^{*}, * *, * * *$ \\
\hline
\end{tabular}

*Statistically significant as compared to control group (group I). ** Statistically significant as compared to group II. *** Statistically significant as compared to group III. 


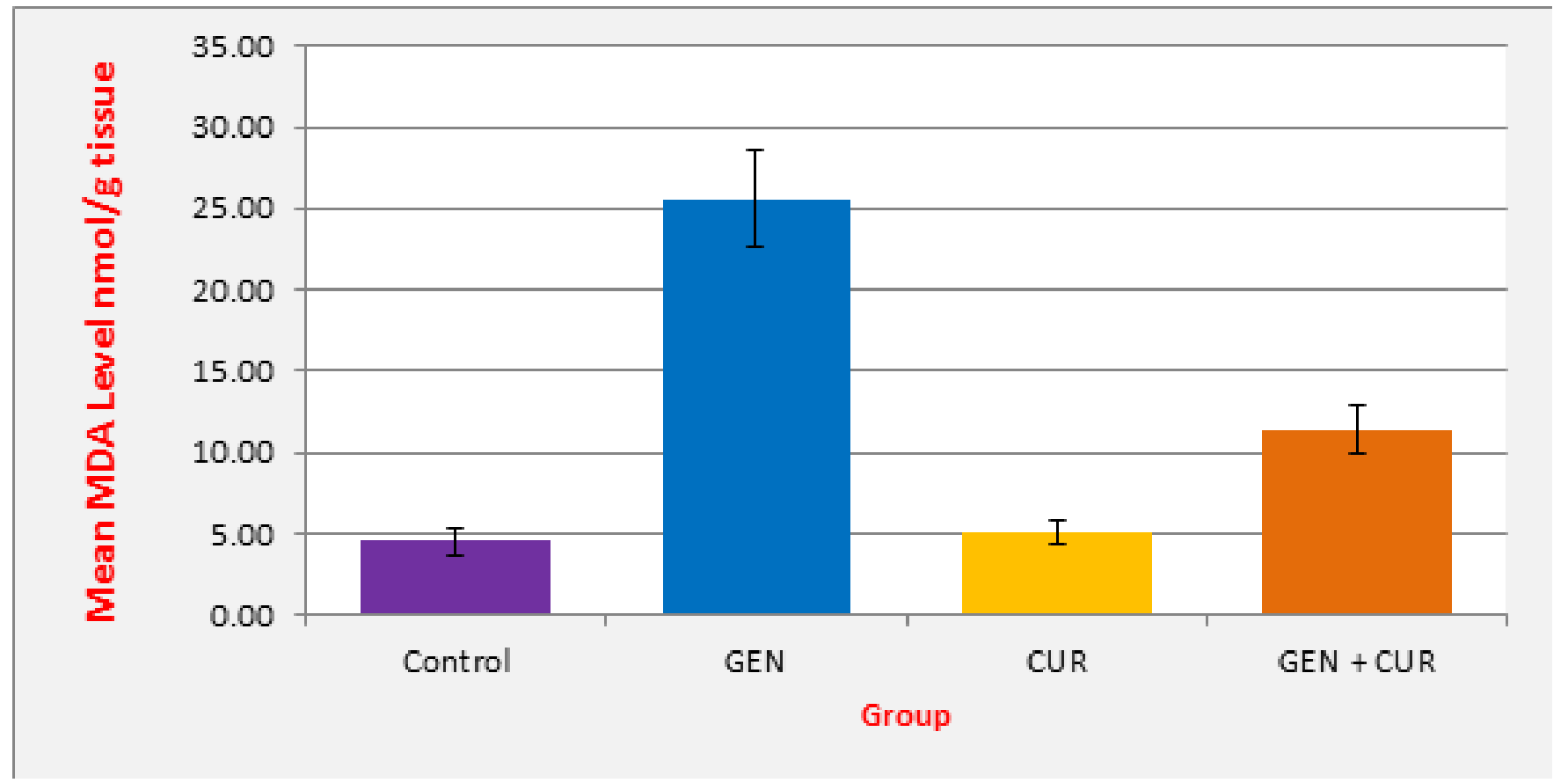

Histogram 4: Comparison of mean MDA level between different groups

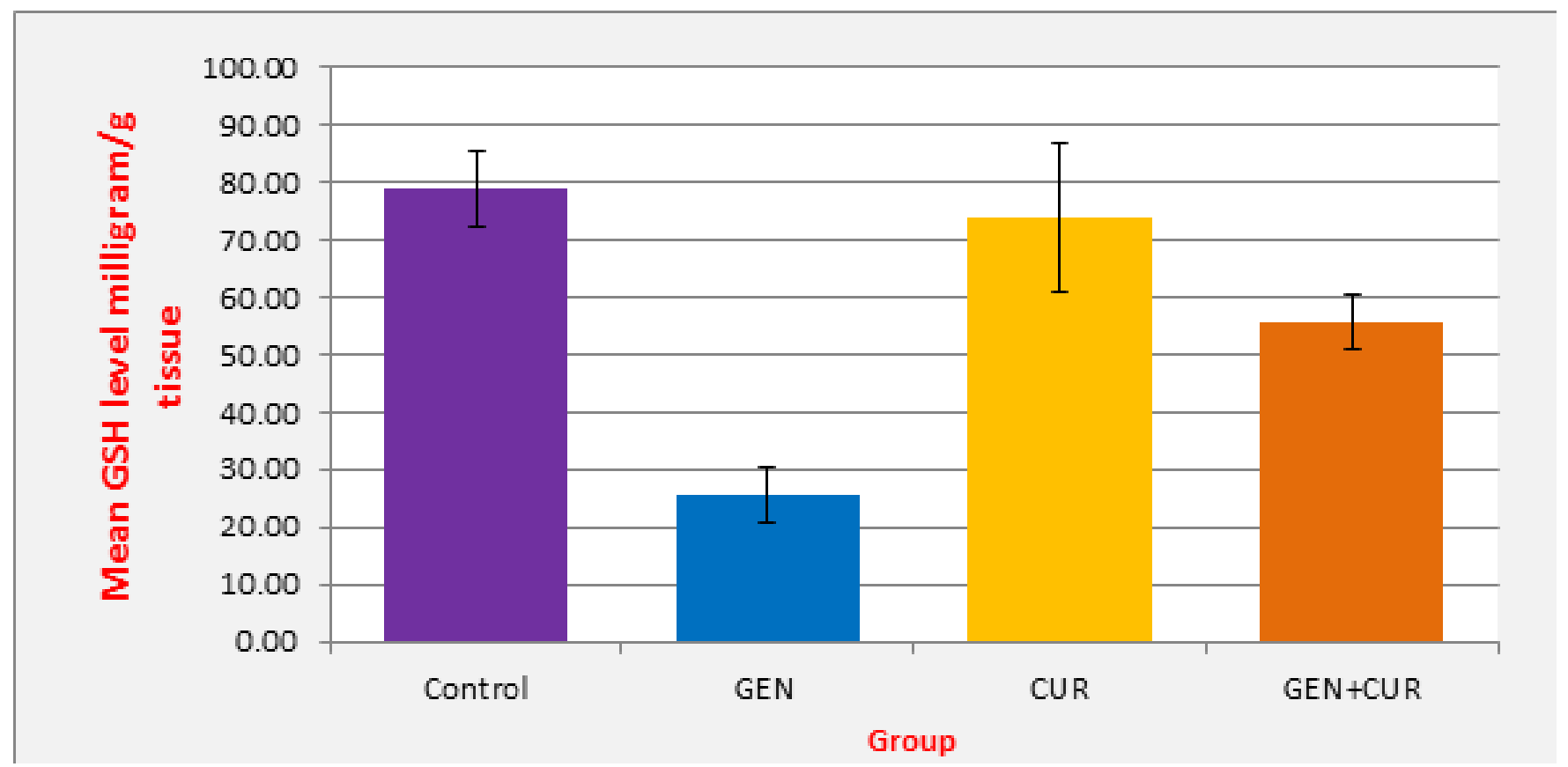

Histogram 5: Comparison of the mean GSH level between different groups

\section{DISCUSSION}

Aminoglycosides are antibiotics which induce many nephrotoxic effects by possible three mechanisms; renal tubular toxicity, reduced glomerular filtration and reduction in renal blood flow ${ }^{[14]}$. Gentamycin is one of the aminoglycosides that is used in the treatment of severe gram-negative infections. However, its nephrotoxicity has limited the use of $\mathrm{it}^{[15]}$. The group of rats treated with gentamicin (GM group) showed pathohistological changes in the form of the enlargement of glomeruli as well as the presence of inflammatory infiltrates in certain areas. The significant changes appeared in the proximal tubules clearly in the form of necrosis and cytoplasm vacuolization of tubular epithelial cells. Less changes appeared in the distal tubule. These data were confirmed by ${ }^{[16]}$ who proved that the proximal tubular cells have receptors that bind to gentamicin and then, invaginated by the cells where it activates lysosomes and mitochondria leading to overproduction of free radicals and by this mechanism, 
gentamycin might produce the histopathological toxicity in this work.

Also, in agreement of this study, ${ }^{[17]}$ reported that gentamyocin affects the kidneys harmfully by the production of reactive oxygen species (ROS) leading to lysosomes resulting in its destabilization and the release of lysosomal enzymes causing cell destruction. Moreover, ${ }^{[18]}$ confirmed this study by giving gentamicin at a dose of $80 \mathrm{mg} / \mathrm{kg}$ to experimental rats that produces significant nephrotoxicity in these rats as evidenced by histological renal changes in the form of tubular necrosis, dilatation of tubules, degen $\neg$ eration of tubular epithelial cells with casts in the tubular lumen, cell infiltration in interstitium, marked congestion of the glomeruli and extensive necrosis. In addition, ${ }^{[19]}$ documented the injurious effects of aminoglycosides affecting the kidneys of numerous species of experimental animals in the form of pathological changes similar to this study. However, ${ }^{[1]}$ reported that the dose has a very important factor in the aminoglycosideinduced nephrotoxicity as giving multiples low doses of aminoglycosides have long been known to cause less pathological alterations in the renal tissue. Furthermore, ${ }^{[20]}$ detected the histopathological changes in distal tubules of the nephron after induction of acute necrosis in proximal tubules by the use of very high doses of gentamicin (400 $\mathrm{mg} / \mathrm{kg}$ for 2 days). Moreover, ${ }^{[21]}$ have been reported that many factors affects the binding of Gentamicin to cell and organelle membranes as the ischemia that enhances this binding and ca2+ that inhibits it competitively. In addition, ${ }^{[22]}$ suggested other mechanisms for Gentamicin nephrotoxicity such as increasing the membrane fluidity of mitochondria, lysosomes and microsomes however, the mechanism of this effect is still unclear

The present study also had quantitatively proved that the proximal tubular epithelium and the glomeruli were actually affected by apoptosis upon exposure to gentamicin. This was confirmed by the significant increase in the area $\%$ of BAX (Apoptotic marker) expression in GEN group. Several studies reported that apoptosis is as a known mechanism of the gentamicin induced nephrotoxicity causing acute tubular necrosis and acute renal failure in 10$15 \%$ of all cases ${ }^{[23]}$. Furthermore, ${ }^{[24]}$ added that the rupture of the lysosomal and mitochondrial membranes releases more apoptotic signals such as acid hydrolases that bind to specific receptors inducing the process of apoptosis and necrosis especially of the cells of the proximal tubules. Moreover, ${ }^{[25]}$ had considered apoptosis as a necessary process in the development of the gentamicin renal pathology by the way of activation of proapoptotic proteins that are responsible for the apoptosis.

This study supported researches done by ${ }^{[26]}$ who found that co-administration of curcumin in animals treated by gentamicin ameliorated the architecture and the pathological changes of the kidney. Curcumin in the current work had attenuated significantly the area $\%$ of
BAX expression confirming its antiapoptotic role. Also, the area $\%$ of PCNA positive reaction was significantly increased in CUR+GEN group as compared to GEN group declaring the cell proliferating promoting role of Curcumin These results are also in agreement with ${ }^{[27]}$ who reported that curcumin has an important prophylactic role through its effective anti-inflammatory and antioxidant actions with inhibition of reactive oxygen species formation. However, ${ }^{[28]}$ has been suggested another mechanism by which curcumin initiates its protective effect through stabilization of the cell membrane by altering the membranes cation-binding characteristics. Further, ${ }^{[24]}$ mentioned that curcumin can decrease tumor necrosis factor (TNF)- $\alpha$ and monocyte chemoattractant protein (MCP) levels in acute nephrotoxicity induced by high doses of gentamicin reducing their inflammatory injurious actions. On the other hand, Tirkey et al., (2005) ${ }^{[7]}$ considered the dose as an important factor in the prophylactic effect of the curcumin as they proved that higher doses of CUR had been associated with more significant improvement of the renal dysfunction in the rat kidney that were checked by the measurement of creatinine clearance and the levels of serum creatinine and BUN which were significantly ameliorated.

The ability of curcumin, in the current work, to induce the cell regeneration of the proximal tubules and glomeruli as observed in group IV rats in comparison to group II rats after being stained by PCNA, was in accordance to Akram et al., (2010) ${ }^{[29]}$ who proved the ability of curcumin to reverse the gentamicin induced nephrotoxicity by its action as a catalyst of the proximal tubules to regenerate and resume its normal architecture. However, Padmini and Kumar (2012) $)^{[30]}$ reported that the recovery and the regeneration of the damaged renal tissues depended on dose and duration of gentamicin administration as they gave an evidence that spontaneous recovery of the injured renal tissues occur faster when use a lower doses of gentamicin with longer duration.

\section{CONCLUSION}

In conclusion, Curcumin could have a protective role against gentamicin adverse renal changes by decreasing apoptosis and promoting cell proliferation.

\section{CONFLICT OF INTEREST}

There is no conflict of interest

\section{REFERENCES}

1. Mingeot-Leclercq M-P. and Tulkens P.M. (1999): Aminoglycosides: nephrotoxicity. Antimicrob Agents Chemother; 43(5):1003- 1012.

2. Ali B.H.(2003): Agents ameliorating or augmenting experimental gentamicin nephrotoxicity: some recent research. Food Chem Toxicol; 41:1447-52. 
3. Eslami S.H., Ebrahimzadeh M.A.,Moghaddam H.A., Nabavi S.F., Jafari N. and Nabavi S.M. (2011): Renoprotective effect of Eryngium caucasicum in gentamicin-induced nephrotoxic mice. Arch Biol Sci; 63 (1):157-160.

4. Kuhad A., Tirkey N., Pilkhwal S. and Chopra K. (2006): Effect of Spirulina, a blue green algae, on gentamicin-induced oxidative stress and renal dysfunction in rats. Fundam Clin Pharmacol; 20:121-8.

5. Koyner J.L., Sher A.R. and Murray P.T. (2008): Antioxidants. Do they have a place in the prevention or therapy of acute kidney injury? Nephron Exp Nephrol; 109:e109-17.

6. Abdel-Raheem I.T., El Sherbiny G.A. and Taye A. (2010): Green tea ameliorates renal oxidative damage induced by gentamicin in rats. Pak $\mathrm{J}$ Pharm Sci; 23:21-8.

7. Tirkey N, Kaur G, Vij G, and Chopra K. (2005): Curcumin a diferuloylmethane, attenuates cyclosporine induced renal dysfunction and oxidative stress in rat kidneys. J. Biosc., 22 (2) 233-46.

8. Ruby AJ, Kuttan G, Babu KD and Kuttan R (2005): Anti-tumour and antioxidant activity of natural curcuminoids. Cancer. Lett., 94:79-83.

9. Karadeniz, A., Yildirim, A., Simsek, N., Kalkan, Y. and Celebi, F. (2008): Spirulina platensis protects against gentamicin-induced nephrotoxicity in rats. Phytotherapy Research 22: 1506-1510.

10. Chuang, S.E., Cheng, A.L., Lin, J.K. and Kuo, M.L. (2000): Inhibition by curcumin of diethylnitrosamine-induced hepatic hyperplasia, inflammation, cellular gene products and cellcycle-related proteins in rats. Food and Chemical Toxicology 38: 991-995.

11. Ohkawa H., Ohishi N. and Yagi K. (1979): Assay for lipid peroxides in animal tissues by thiobarbituric acid reaction. Analytical Biochemistry 95(2): 351-358.

12. Aebi H (1984): Catalase in vitro. Methods in Enzymology 105: 121-126.

13. Sanderson S, Wild G, Cull AM, Marston J, Zardin G. Immunohistochemical and immunofluorescent techniques. In: Suvarna SK, Layton C, Bancroft, JD, Editors. Bancroft's Theory and Practice of Histological Techniques, Eighth Edition, Elsevier Limited, 2019: 337-394.
14. WargoK.A. andEdwards J.D. (2014): Aminoglycosideinduced nephrotoxicity.J Pharm. Pract.; 27:573-577.

15. Nakas-Ic'indic' E., Avdagic' N. and Mijanovic et al., (2005): Nitric oxide in gentamicin-induced acute tubular necrosis in rats. Bosn J Basic Med Sci.; 5:70-74.

16. Stojiljkovic N., Stoiljkovic M. and Mihailovic D et al., (2012): Beneficial effects of calcium oral coadministration in gentamicin-induced nephrotoxicity in rats. Ren Fail; 34:622627.

17. Cuzzocrea S., Mazzon E. and Dugo et al., (2002): A role for superoxide in gentamicin-mediated nephropathy in rats. Eur J Pharmacol.

18. Nair Aiswarya, Rao Rashmi R, Shenoy Preethi J, Vinod Chandran, S Teerthanath, Pai Sunil B, Rakesh K.B. (2018): Nephroprotective Effect of Aqueous Extract of Pimpinella anisum in Gentamicin Induced Nephrotoxicity in Wistar Rats. Pharmacogn J. 2018; 10(3):403-407.

19. Istrate C., Mingeot-Leclercq M.-P. and Moisescu M.-G. (2012): Interaction of gentamicin polycation with model and cell membranes. Bioelectrochemistry; 87:230-235.

20. Nouwen E. J., Verstrepen W. A., Buyssens N., Zhu M.-Q. and De Broe M. E. (1994): Hyperplasia, hypertrophy, and phenotypic alterations in the distal nephron after acute proximal tubular injury in the rat. Lab. Investig.70:479-493.

21. Dhanarajan R, Abraham P and Isaac B (2006): Protective Effect of Ebselen drug, against gentamicin-induced renal damage in Rats, Basic. Clin. Pharmacol. Toxicol., 99(3), 267-72.

22. Shimmi S.C., Jahan N., Baqi N. and Rahman Z. (2014): Histological evidence of nephroprotective effect of Ashwagandha (Withania somnifera) root extract against gentamicin induced nephrotoxicity in rats. J Enam Med Col.; 4(1):26-30

23. Jovanovic D., Jovovic D., Mihailovic N. and Maksic N. (2005): Influence of carvedilolon on chronic renal failure progression in spontaneously hypertensive rats with adriamycin nephropathy. Clin. Nephrol., 63(6)446-53.

24. Servais, H.; Van Der Smissen, P.; Thirion, G.; Van der Essen, G.; Van Bambeke, F.; Tulkens, P. M. \& Mingeot-Leclercq, M. P. (2005):Gentamicininduced apoptosis in LLCPK1 cells: involvement of lysosomes and mitochondria. Toxicol. Appl. Pharmacol., 206(3):321-33. 
25. Han, S. Y.; Chang, E. J.; Choi, H. J.; Kwak, C. S.; Park, S. B.; Kim, H. C. \& Mun, K. C. (2006): Apoptosis by cyclosporine in mesangial cells. Transplant. Proc., 38(7):2244-6.

26. Manikanadan R, Beulaja $M$, Thiagarajan $R$, Priyadarsini A, Saravanan R and Arumugam M (2011): Ameliorative effects of curcumin against renal injuries mediated by inducible nitric oxide synthase and nuclear factor kappa B during gentamicin-induced toxicity in wister rats. Eur $\mathbf{J}$ Pharm; 670: 578-585.

27. Biswas SK, McClure D, Jimenez LA, Megson IL, Rahman I (2005): Curcumin induces glutathione biosynthesis and inhibit NF-kappa B activation and interleukin-8 release in alveolar epithelial cells. Mechanism of free radical scavenging activity. Anti Red Sign; 7: 32-41.
28. Venkatesan N, Durairaj P and Arumugam (2000): Curcumin prevents adriamycin neph-rotoxicity in rats. Br. J. Pharmacol., 129, 231-4.

29. Akram M, Shahab-uddin, Ahemed A, Khan U. (2010): Curcuma longa and curcumin: A review article. Rom J Biol; 55(2):65-70.

30. Padmini M.P., Kumar J.V., (2012): A histopathological study on gentamicin induced nephrotoxicity in experimental albino rats. IOSR; 1:14-7. 